\title{
Acción social municipal en situaciones de urgencia y emergencia social. Experiencia de las capitales de la CAPV
}

\section{Txema Duque}

Ayuntamiento de Bilbao

$<$ txema.duque@ayto.bilbao.net>

\section{Iñigo Estomba}

Ayuntamiento de Donostia-San Sebastián

\section{Joxanjel Agirre}

Ayuntamiento de Vitoria-Gasteiz
Artikulu honetan, euskal udaletako gizarte-zerbitzuen gizarte-larrialdi egoerak artatzeko esku-hartzeen berri ematen da. Horretarako, berrikusi dituzte aro demokratikoan hiru euskal hiriburuetan garatutako esperientziak -Bilbo, Donostia eta Gasteiz-. Erabateko egoera berri bat ekarri zuen Euskal Autonomia Erkidegoko Gizarte Zerbitzuei buruzko 12/2008 Legeak. Izan ere, gizartelarrialdiko egoera horiek gizarte-zerbitzuen esku jartzen ditu legeak, eta Prestazioen eta Zerbitzuen Katalogoak kontuan hartzen ditu gizartelarritasunen zerbitzu horiek.

\section{HITZ-GAKOAK:}

gizarte-larritasuna, premia, gizarte-zerbitzuak, lehen-mailako arreta, zerbitzu-zorroa.
En este artículo se explica la intervención de los servicios sociales municipales en situaciones de urgencia y emergencia social. Para ello, se repasa la experiencia desarrollada en las tres capitales vascas -Bilbao, Donostia-San Sebastián y Vitoria-Gasteizen la etapa democrática. La Ley 12/2008, de Servicios Sociales, de la CAPV abre un nuevo escenario, en el que la situación de emergencia es un ámbito de intervención del sistema público de servicios sociales, y en el que el servicio de urgencias está contemplado en el Catálogo de Prestaciones y Servicios.

\section{PALABRAS Clave:}

emergencia social, urgencia, servicios sociales, atención primaria, cartera de servicios. 


\section{Introducción}

En esta breve exposición, trataremos de explicar la respuesta que ofrecemos desde los servicios sociales municipales de las tres capitales vascas a las personas que se encuentran en una situación considerada de emergencia. Para ello, echaremos un vistazo a nuestra historia, a cómo empezamos, a de qué manera se han ido desarrollando los servicios, y a qué conclusiones y cuestiones nos parecen importantes para seguir avanzando. Cada ciudad es una realidad distinta, por ello, nos encontramos con tres respuestas diversas. En todo caso, valoramos muy positivamente el intercambio responsable de experiencias (análisis, programas, estrategias, documentación) y la colaboración mutua existente. En realidad, se trata de una reflexión conjunta sintetiza la evolución de nuestros servicios sociales de urgencia durante los últimos quince años.

\section{Marco normativo}

La intervención de nuestros servicios sociales municipales en situaciones de urgencia y emergencia social se ha realizado de la mano de las tres leyes básicas sobre servicios sociales que hemos tenido en la CAPV. Como exponemos a continuación, la ley de 1996 planteó expresamente los 'servicios de acogimiento de urgencia', regulación ampliada en el Decreto 155/2001. A pesar de ello, podemos decir que resultó insuficiente y ambigua en cuanto a contenidos y conceptos, $y$, por otro lado, que fue mayoritariamente incumplida por parte de los municipios obligados a disponer de dichos servicios.

Por su parte, nuestra primera Ley, la 6/82, únicamente incluyó en su artículo 3, sobre áreas de actuación, "la colaboración con los organismos competentes en situaciones de emergencia social". En este contexto, con motivo de las inundaciones de agosto de 1983, en el Ayuntamiento de Bilbao, que disponía de unos mínimos servicios sociales, se aprovechó para su primer desarrollo y el posterior lanzamiento de la etapa democrática.

\subsection{Ley $5 / 96$, de Servicios Sociales de la CAPV}

Sustituye a la Ley 6/82, de Servicios Sociales y tiene como objeto fundamental ordenar, estructurar, promover y garantizar, mediante un sistema de responsabilidad pública, el derecho de la ciudadanía a los servicios sociales, con el fin de:

- Prevenir y eliminar las causas de marginación social y de desigualdad.

- Promover la integración social de las personas y colectivos.

- Favorecer el pleno y libre desarrollo de las personas y colectivos.
En su art. 12.2, se dice que:

En todos los municipios con población superior a 20.000 habitantes, además del Servicio social de Base, existirán servicios destinados al acogimiento de urgencia para atender situaciones críticas determinadas por la carencia de alojamiento o por graves conflictos convivenciales, así como centros que favorezcan la convivencia social y la solidaridad entre los/las ciudadanos/as.

\subsection{Decreto 155/2001, de Determinación de Funcio- nes en Materia de Servicios Sociales}

Sobre las funciones de los ayuntamientos en referencia a los programas y servicios enmarcados en el artículo 12.2 de la Ley 5/1996, de Servicios Sociales, su artículo 4.2.1 realiza las siguientes matizaciones en torno al llamado 'servicio de acogimiento de urgencia':

- El servicio de acogimiento de urgencia irá dirigido a atender situaciones críticas determinadas por la carencia de alojamiento, carencia que podrá deberse bien a la ocurrencia de un siniestro en la vivienda, bien a carencias socio-económicas, bien a graves conflictos convivenciales, que pongan en peligro la seguridad física o emocional de las personas que solicitan la intervención del servicio.

- La función de este servicio de acogimiento de urgencia consistirá en ofrecer una solución residencial de carácter temporal a las personas afectadas, poniendo su caso en conocimiento del servicio social de base, para que éste se encargue de informar, valorar y orientar el caso.

- El servicio de acogimiento de urgencia se puede articular concertando plazas hoteleras en el municipio de residencia, en pisos de acogida específicos, que garanticen la seguridad física 0 emocional de las personas, así como la confidencialidad de su ubicación, o bien en albergues, destinados a personas que carecen de domicilio fijo.

\subsection{Ley $12 / 2008$, de Servicios Sociales del País Vasco}

En el artículo 3, sobre los titulares del derecho a los servicios sociales, dice que:

[...] las personas que se encuentren en la Comunidad Autónoma del País Vasco podrán acceder, en todo caso, al servicio de información, valoración, diagnóstico y orientación, así como al acompañamiento social, y a aquellos servicios y prestaciones que sean definidos como de urgen- 
cia social en la Cartera de Prestaciones y Servicios de Sistema Vasco de Servicios Sociales.

En el artículo 6, sobre la finalidad del Sistema Vasco de Servicios Sociales, encontramos que los fines del sistema son "prevenir y atender las necesidades personales y familiares originadas por las situaciones de dependencia, desprotección y exclusión así como las provocadas por las situaciones de emergencia”.

Sobre las prestaciones económicas, el artículo 16 menciona "las prestaciones para facilitar la integración social y/o la autonomía así como para cubrir o paliar las consecuencias económicas de las situaciones de emergencia o urgencia social, dependencia 0 desprotección". Este servicio se recoge como tal en el artículo 22, apartado 3.1.

El Catálogo de Prestaciones y Servicios, en su artículo 22, apartado 2.6, recoge el 'servicio de coordinación a urgencias sociales'.

Sobre la organización y estructura del Sistema Vasco de Servicios Sociales, el artículo 27.2a expone que los servicios sociales de atención primaria, regulados en el artículo 22.1,

[...] posibilitarán el acceso de las usuarias y usuarios al conjunto del Sistema de Servicios Sociales y atenderán las necesidades relacionadas con la autonomía, la inclusión social y las situaciones de urgencia o desprotección social, con particular incidencia en la prevención de las situaciones de riesgo.

En la disposición adicional séptima, sobre reserva de plazas para las urgencias sociales se dice textualmente que

Las administraciones públicas vascas deberán, en los servicios de su competencia, garantizar una reserva de plazas a efectos de dar respuesta a las demandas derivadas desde los servicios de urgencias sociales y desde los servicios de respiro.

\section{Nuestra historia: origen y evolución}

Los elementos presentes en el origen de un servicio no sólo son significativos en la primera etapa, sino que condicionan, de alguna manera, el desarrollo posterior del citado servicio. Encontramos algunos elementos que han tenido su importancia en el origen de nuestros servicios de urgencias (puede variar el grado de ponderación en cada municipio) y que exponemos de forma genérica:

- El contexto y la realidad de la red de servicios sociales municipales del momento. Características como el dimensionamiento, el personal o la situación de la lista de espera han tenido su influencia.

- Percepción de las situaciones de desprotección por parte de la ciudadanía, tolerancia en la convivencia con esas situaciones y papel de los agentes sociales, los técnicos y los partidos políticos.

- Oportunidad y pertinencia (coyuntura) desde el punto de vista político (elecciones) y presupuestario (económico).

- Conciencia de la necesidad de realizar actuaciones coordinadas entre diversas áreas municipales, partiendo de criterios de colaboración y respeto de competencias (entre dos o más áreas).

- Experiencia de colaboración no protocolizada en emergencias ordinarias (incendios, derrumbes, explosiones, accidentes).

- Experiencia de grandes catástrofes (inundaciones en Bilbao 1983, camping de Biescas, 11-M).

- Decisión política: responsabilidad pública de ofrecer un servicio integral a las personas que se encuentran en una situación de emergencia.

\subsection{Vitoria-Gasteiz}

El Ayuntamiento de Vitoria-Gasteiz pone en marcha el Servicio Municipal de Urgencias Sociales en 1988. En un comienzo, se trata de un servicio 'de gestión indirecta', convenido con la Cruz Roja; existía, además, un trabajador municipal que atendía a las personas domiciliadas de la ciudad para coordinar su alojamiento en el Centro Municipal de Acogida Social.

Posteriormente, en 1992, dicho servicio se municipaliza, pasando las trabajadoras sociales a depender directamente de la administración municipal. En 1996, vuelve a ser gestionado por una entidad externa, hasta la fecha. Actualmente, son once las profesionales que desarrollan su trabajo en el servicio: una coordinadora, nueve trabajadoras sociales y una psicóloga.

El marco de la actuación y los objetivos de este servicio son:

- Atención social de primera acogida en situaciones de crisis de aquellas personas que lo precisen, durante las 24 horas del día, los 365 días del año.

- Facilitar a los/as ciudadanos/as que lo demanden información sobre los recursos sociales existentes en el territorio histórico de Álava, y aplicación de los recursos (centros y programas) de que dispone la red de servicios sociales municipales.

- Atender de forma inmediata las llamadas de los/as ciudadanos/as de Vitoria-Gasteiz al teléfo- 
no 010 cuando sean de contenido social y derivarlas al Departamento de Intervención Social. Asimismo, atenderá las necesidades sociales detectadas por otros departamentos municipales, la Policía Local, SOS Deiak, el sistema sanitario u otros agentes sociales, y derivadas al servicio.

- Intervención en situaciones de emergencia social: incendios, derrumbamientos, accidentes de circulación, inundaciones u otros siniestros.

- Atención social a las personas transeúntes e indomiciliadas, 'sin techo', colectivos nómadas e itinerantes: oferta de acogida e ingreso, mediante derivación, al Centro Municipal de Acogida Social, a la Casa Abierta, al Centro de Noche Aterpe y a otros recursos de acogida existentes, o que surjan en el futuro, en este ámbito de actuación.

- Atención y seguimiento de colectivos de alto ries go de marginación social, o en situación extrema de exclusión social, residentes en el municipio: personas drogodependientes en programas de tratamiento y que carecen de entorno familiar, personas detenidas por la Policía Municipal, personas enfermas de sida, personas y familias extranjeras en situación de irregularidad documental, entre otras.

- Aplicación de los recursos sociales (programas y servicios) de que dispone el Departamento Municipal de Intervención Social para solventar las necesidades de las personas que se atiendan en el servicio.

- Coordinar las intervenciones con otros servicios propios y ajenos al Ayuntamiento, globalizar y analizar las demandas sociales recibidas para mejorar la eficacia y la eficiencia de las actuaciones sociales, elevando las oportunas propuestas al órgano correspondiente.

- Servir de observatorio social: realizar prospecciones para prevenir necesidades sociales emergentes.

Además, se ha trabajado y actualizado el Protocolo de Atención a la Demanda Urgente, que es el que marca las funciones del Servicio Municipal de Urgencias Sociales en el Departamento Municipal de Intervención Social, y lo que ello implica: procedimientos de derivación, coordinación y acompañamiento con otros equipos y servicios, por ejemplo.

\subsection{Bilbao}

El Ayuntamiento de Bilbao inicia el Servicio Municipal de Urgencias Sociales en junio de 1999. Hasta 2007, mantiene su ubicación en el Albergue Municipal de Elejabarri. Durante esos años, desarrolla los siguientes programas:
- Programa de Atención a Situaciones de Urgencia y Emergencia.

- Programa de Corta Estancia (de entre uno y siete días) en el citado albergue.

- Programa de Incorporación Social, con dos pisos de un total de nueve plazas.

A partir de marzo de 2007 , se traslada al centro de Bilbao (Mazarredo, 22), y en esta nueva etapa, gestiona dos programas:

- Programa de Intervención en Situaciones de Emergencia y Urgencia Social en el municipio. Es la razón de ser del servicio y actúa en coordinación directa con los departamentos municipales de Policía Municipal, Bomberos y Ambulancias, así como con los Servicios Sociales de Base.

- Programa de Acceso a Prestaciones Elementales (alojamiento temporal, alimento y vestido) para personas del municipio y de paso, siendo este programa la referencia única para personas usuarias, profesionales y ciudadanía en general. Se trata de un sistema de gestión e información compartida, disponible en tiempo real, centralizado e informatizado. Los protocolos para el diagnóstico y la aplicación de prestaciones son:

- Plazas de alojamiento: reservas en el Albergue Municipal de Elejabarri, el Centro de Noche Hontza, el Centro de Noche Lagun Artean, el Dispositivo de Alojamiento Invernal y el Centro de Noche de Baja Exigencia.

- Plazas de comedores sociales: reservas de menú cargadas en carné individual de lectura óptica, en Apostólicas, Franciscanos y Conde de Aresti.

- Vestido: pedidos de ropa en tiendas de Berohi y Emaús.

En 2008, la empresa Aurrerantz -adjudicataria del contrato administrativo del Servicio Municipal de Urgencias Sociales- obtiene la certificación de su sistema de gestión de la calidad, según la norma ISO 9001/2000, para los dos programas citados anteriormente. El servicio lo componen nueve trabajadoras sociales, una administrativa y una coordinadora.

En 2010, se elabora la Carta de Servicios sobre la Intervención Municipal en situaciones de Emergencias Ordinarias, en la que nuestro servicio juega un papel central en el proceso de atención a personas afectadas. A principios de 2011, se realiza el Procedimiento de Actuación Coordinada entre el servicio de urgencia y los servicios sociales de base. 


\subsection{Donostia-San Sebastián}

El Ayuntamiento de Donostia-San Sebastián inicia el Servicio Municipal de Urgencias Sociales en mayo de 2007. Sus principales características son: la profesionalidad (se trata de trabajadoras con formación técnica suficiente y formadas), la disponibilidad (deben poder actuar en muy poco espacio de tiempo, sin que ello suponga un perjuicio grave para terceros) y la movilidad (deben poder personarse en cualquier lugar dentro del término municipal). Atendiendo a estas características, se han establecido cuatro funciones diferenciadas, dentro de la Dirección de Bienestar Social, que son:

- Atención inmediata a situaciones de urgencia, que requieren una valoración y, en su caso, una respuesta inmediata. Esta función es la que da nombre al servicio y, quizá, representa su esencia, pero no es necesariamente la que supone mayor dedicación.

- Gestión de recursos sociales, tales como el Neguko Aterpea, el Servicio de Puertas Abiertas, los pisos de emergencia, el Centro para Familias o el acceso a medicamentos.

- Responsabilidad de caso. Las técnicas del servicio son responsables de casos de personas que no tienen una ubicación clara en el territorio del municipio (personas sin hogar) o de casos extremadamente demandantes que interfieren excesivamente en el funcionamiento, menos flexible, de los centros de servicios sociales. A estos efectos, sus obligaciones se equiparan a las de las trabajadoras sociales de cualquier otro de esos centros (diagnóstico, coordinación, establecimiento del plan individual de actuación).

- Apoyos puntuales y acompañamiento. Debido a la mayor disponibilidad y movilidad -recuérdese que son, junto con la capacidad técnica, sus características definitorias-, el Servicio Municipal de Urgencias Sociales realiza funciones de apoyo puntual a demanda de los/las profesionales responsables de caso de otros servicios del Departamento.

\section{4. Ámbito de actuación (situaciones de urgencia/emergencia)}

Es un debate muy habitual, esto quiere decir que sigue abierto. Sin pretender agotarlo, ofrecemos algunas aportaciones:

- No existe consenso general suficiente en relación a los términos de urgencia y emergencia en el ámbito de lo acción social.

- Emergencia: se utiliza en el ámbito de la Protección Civil, está ligada a la ocurrencia de un acci- dente y hace relación a una situación definida como multidimensional y que, por lo tanto, precisa un abordaje interdisciplinar (integral).

- Urgencia: se utiliza en el ámbito sanitario y de los servicios sociales ${ }^{1}$. Parece más ligado directamente al indicador tiempo de respuesta, que viene marcado por el grado de necesidad o nivel de gravedad. En el plano operativo, la priorización se determinará según una valoración del riesgo.

- En el ámbito sanitario, emergencia está relacionada con riesgo vital, y urgencia, con situaciones en las que no se vería comprometida la integridad personal. En otros ámbitos, urgencia social se aplica cuando afecta a una persona o familia, y emergencia social, cuando afecta a un colectivo, distinguiéndola de la gran emergencia o catástrofe.

No es que el debate esté falto de sentido, pero lo cierto es que, en estos momentos, se manifiesta como improductivo. Con objeto de superar esta situación de cierto bloqueo conceptual -no de agotar, ni evitar seguir profundizando en ella-, nos proponemos utilizar de forma indistinta los términos urgencia y emergencia. En ambos casos, aceptamos como imprescindible la necesidad de atención inmediata e ineludible por parte de los servicios sociales.

Podemos denominar urgencia/emergencia social a esa situación producida por un hecho no previsto, debido generalmente a causas naturales o provocadas (riesgos tecnológicos y antrópicos), que afecta y compromete las capacidades personales, los recursos y medios de subsistencia, así como las relaciones o redes sociales y familiares. Las personas afectadas se encuentran en una situación de desprotección grave; por ello, precisan una intervención inmediata e ineludible, para evitar que esta situación se agrave o genere mayor perjuicio -quizás irreversible- a la persona afectada.

Así, nos encontramos con urgencias/emergencias sociales que afectan a una persona o familia (emergencia individual); otras veces, se ven afectadas varias familias (emergencias colectivas); y en ocasiones, una gran parte de la población (grandes emergencias o catástrofes).

Las situaciones de urgencia/emergencia social, en muchos casos, se constituyen, en sí mismas, en un elemento emergente de estados de riesgo o exclusión social. Por tanto, visibilizan situaciones de necesidad que permanecían ocultas. Las situaciones

${ }^{1}$ En este sentido, el sistema de servicios sociales, al igual que otros sistemas, como el sanitario, dispone de tres modalidades de atención a personas usuarias: ordinaria (con cita previa), preferente o prioritaria (relacionada con plazos) y urgente (inmediata, en el servicio específico de urgencias). 
de emergencia suponen igualmente un factor de riesgo en personas en situación previa de vulnerabilidad social.

En esta exposición no nos fijamos en las denominadas grandes emergencias, sino que ponemos el foco especialmente en esas situaciones a las que hacemos frente con los recursos humanos y materiales ordinarios destinados habitualmente para ello (servicio de urgencias). Casi siempre actuaremos con la Policía Municipal y, con frecuencia, también intervendrán sanitarios y bomberos. Por lo tanto, se puede decir que nos vamos a encontrar con:

- Sucesos imprevistos precipitantes en la vida de los sujetos o de la colectividad.

- Sucesos que afectan y comprometen las capacidades, los recursos y los medios de subsistencia, así como las relaciones o redes familiares y sociales.

- Situaciones de vulnerabilidad, fragilidad, impotencia, desamparo.

- Necesidad de contención y acompañamiento psicosocial.

- Necesidad de proporcionar una respuesta rápida y cercana al ciudadano.

- Situaciones donde se movilizan recursos normalizados o específicos con criterios de urgencia.

- Respuestas de carácter temporal y transitorio.

En estas situaciones, los servicios sociales actúan en coordinación con otros servicios intervinientes: sanitarios, bomberos, Policía Municipal.

El objeto de la intervención de los servicios sociales es procurar el bienestar de las personas afectadas a través de un adecuado diagnóstico de las situaciones de las personas, teniendo en cuenta sus necesidades, sus posibilidades (capacidades) y los recursos formales e informales disponibles para garantizar su bienestar biopsicosocial.

A partir de la experiencia de estos años, describimos a continuación las situaciones genéricas ${ }^{2}$ (evitamos hacerlo por colectivos) que entendemos pueden ser catalogadas como de urgencia/emergencia y que serán objeto de intervención inmediata por parte del Servicio Municipal de Urgencias Sociales -previo aviso de la Policía Municipal-:

- Situaciones de pérdida y/o desorientación: se refiere a esas situaciones cuya característica principal es que la persona se encuentra perdida o desorientada, y precisa ayuda profesional

2 Estas tres primeras situaciones han sido protocolizadas por el SAMUR Social del Ayuntamiento de Madrid. inmediata. Pueden afectar a personas mayores, menores, discapacitadas psíquicas, enfermos mentales, personas sin techo, entre otras.

- Situaciones de desprotección y/o abandono: se refiere a esas situaciones cuya característica principal es que la persona se encuentra abandonada y precisa ayuda profesional inmediata. Pueden afectar a personas mayores, menores (mendicidad, detención de progenitores), discapacitadas psíquicas, enfermos mentales o personas sin techo, por ejemplo.

- Situaciones de soledad sobrevenida: se refiere a situaciones en las que las personas dependientes cuentan con una red de apoyo familiar y social estable, $y$, por un acontecimiento imprevisto, ese apoyo desaparece y la persona dependiente se encuentra sola. Por ejemplo, en el caso de una persona mayor con alzhéimer cuidada por su marido o hijo, cuando éste tiene un accidente, o es retenido por la Policía, y la persona mayor queda sola, o también en fallecimientos repentinos de cuidadores únicos.

- Situaciones de violencia de género: se refiere a esas situaciones cuya característica principal es que la persona es víctima de violencia de género. Se tendrá como referencia el protocolo municipal de coordinación para la atención integral en situaciones de violencia de género entre las áreas competentes.

- Situaciones de emergencia ordinarias o grandes emergencias: son aquellas situaciones provocadas por un hecho imprevisible, debido a causas naturales o provocadas que afecta a un conjunto de personas o colectividad. Se refiere a incendios, accidentes, derrumbamientos, inundaciones, explosiones u otros episodios de naturaleza similar.

\section{Qué hacemos (naturaleza de la intervención)}

En el proceso de intervención caracterizado por la necesidad de atención inmediata e ineludible, realizamos un diagnóstico de necesidades y una aplicación de los recursos disponibles.

\subsection{Necesidades}

Los problemas y necesidades más habituales que detectamos en una situación de urgencia/emergencia social son los siguientes:

- Pérdida de seres queridos.

- Pérdida temporal o definitiva de la vivienda.

- Higiene básica.

- Vestido y calzado. 
- Alimento y agua.

- Imposibilidad de acudir al trabajo y a centros escolares.

- Pérdida de medios económicos.

- Soledad sobrevenida.

- Información.

- Contacto con familiares y amigos.

- Desaparición de personas.

\subsection{Prestaciones}

En relación con las necesidades citadas, los servicios sociales desarrollamos las siguientes funciones:

- Información relacionada con el suceso, el procedimiento que se ha de seguir y los recursos disponibles.

- Orientación y apoyo psicosocial.

- Cobertura de necesidades básicas: alojamiento, alimento, vestido e higiene.

- Gestiones para la localización, el agrupamiento de familiares y el contacto con su red social.

- Gestiones para resolver situaciones personales: medicación, centros escolares, animales de compañía.

- Traslado a espacios de alojamiento inmediato o centros de acogida.

- Localización de familiares.

- Comunicación de malas noticias.

- Tramitación de prestaciones económicas.

- Gestión de recursos de alojamiento alternativo.

- Acompañamiento físico para resolver situaciones personales.

\section{Cómo lo hacemos}

Exponemos a continuación algunos elementos que consideramos fundamentales en relación a la naturaleza, identidad, metodología y organización de nuestros servicios.

\subsection{Responsabilidad pública, provisión del servicio y organización}

Se trata de un servicio de titularidad pública. En las tres ciudades se realiza a través de un contrato con empresas del sector de los servicios sociales. Se presta atención durante 24 horas, los 365 días del año. El servicio está organizado en turnos de maña- na, tarde y noche. Los turnos de mañana y tarde son presenciales, y el turno de noche, localizado. Los perfiles profesionales presentes son: auxiliar administrativo/a, trabajador/a social y psicólogo/a. Un profesional coordina el equipo.

\subsection{Características y metodología básica}

El Servicio Municipal de Urgencias Sociales forma parte de la red de servicios sociales municipales y se encuentra especialmente coordinado con los servicios sociales de base. Dispone de formación y capacidad operativa suficiente, y en concreto:

- Disponibilidad inmediata para la intervención social. Activación, desplazamiento y atención in situ.

- Capacidad de detección y diagnóstico avanzado de necesidades, sujeto a la posterior comprobación de datos.

- Aplicación de recursos sociales de forma inmediata.

- Derivación a los servicios correspondientes para continuar la intervención realizada.

- Registro de la intervención realizada y, en su caso, informes específicos.

\subsection{La coordinación como factor estratégico}

Ningún servicio de urgencias sociales podría gestionar directamente recursos suficientes para realizar por sí mismo e íntegramente todas estas funciones. La coordinación con otros agentes es imprescindible. De hecho, somos la parte de un sistema atención a las urgencias que evalúa las necesidades sociales en una situación de urgencia/emergencia y que tiene capacidad para activar recursos que les den respuesta, de la misma manera que otros evalúan y dan respuesta a los déficits de seguridad física o sanitaria.

Sobre todo en la primera fase de la intervención de urgencias, en la que lo principal es hacer un buen diagnóstico de las necesidades sociales de las personas implicadas, es imprescindible una buena coordinación con los otros servicios de atención de urgencias (bomberos, policías, sanitarios) y sus coordinadores (SOS Deiak, Centros Avanzados). De la misma manera que un profesional de nuestro servicio debe conocer cuándo tiene que llamar a la Guardia Municipal y qué puede esperar de ella, cualquier profesional de atención a las emergencias debería saber cuándo debe llamar a un servicio de urgencias sociales y en qué puede ayudar (qué situaciones personales puede abordar). Esta cuestión, que parece baladí, tiene mucha importancia. Por desgracia, es muy frecuente que, desconociendo 
qué podemos y qué debemos hacer, se llame a los servicios de urgencias sociales sólo cuando ya no se sabe qué hacer, en vez de contar con ellos como un aliado permanente.

Posteriormente, cuando lo principal es dar respuesta a esas necesidades identificadas, se hace imprescindible una coordinación fluida con otros agentes, como los servicios sociales especializados, para que, por un lado, presten un servicio muy orientado a una necesidad o colectivo concreto y, por otra, descongestionen los servicios de acogimiento de emergencia. Así, por ejemplo, necesitamos que las residencias de personas dependientes estén en situación de acoger con inmediatez a una persona que necesite atención permanente en caso de tener que desalojarla de su entorno habitual.

Y, por supuesto, también hay que coordinarse cuando todavía no ha pasado nada. De hecho, el tiempo invertido en determinar con claridad qué tiene que hacer cada quién, en establecer buenos protocolos, en conseguir que los conozcan todos los que deban hacerlo, en detectar posibles problemas antes de que se produzcan, o en anticipar soluciones va a ser tiempo que nos ahorramos en

\subsection{Protocolos de actuación}

Enumeramos una serie de protocolos que, de hecho, están marcando las pautas de nuestras intervenciones.

\subsubsection{Vitoria-Gasteiz}

- Programa de Acceso al Centro Municipal de Acogida Social.

- Protocolo de Actuación Integral en Situaciones de Violencia de Género.

- Protocolo de Actuación para el Dispositivo de Alojamiento Invernal.

- Protocolo de Atención a la Demanda Urgente.

- Protocolo para la Atención a Mujeres Víctimas de Maltrato.

- Protocolo de Nevadas.

- Protocolo de Situaciones de Emergencia.

- Protocolo de Asentamientos Ilegales en el Municipio de Vitoria-Gasteiz (anteriormente, Protocolo de Asentamientos Rodados [Parcela de Agirrelanda]).

- Protocolo de Alarmas en los Recursos de Alojamiento del Servicio de Inserción.

\subsubsection{Bilbao}

- Protocolo de Actuación Coordinada entre el Servicio Municipal de Urgencias Sociales y la Policía Municipal.

- Proceso de Actuación en Emergencias Ordinarias y Grandes Emergencias (carta de servicios).

- Protocolos del Programa de Acceso a Prestaciones Elementales (alojamiento, alimento y vestido).

- Protocolo de Actuación Integral en Situaciones de Violencia de Género.

- Protocolo de Actuación para la Atención de Menores Extranjeros no Acompañados.

- Protocolo de Actuación para el Dispositivo de Alojamiento Invernal.

- Protocolo de Intervención Coordinada Servicio Municipal de Urgencias Sociales-Servicios Sociales de Base.

- Protocolo de Acceso de Urgencia al Programa de Convalecencia para Personas sin Techo.

\subsubsection{Donostia-San Sebastián}

- Protocolo de Actuación ante Situaciones de Urgencia Detectadas por el Servicio de Asistencia Domiciliaria.

- Protocolo de Acceso al Dispositivo de Alojamiento Invernal.

- Protocolo de Comunicación de Malas Noticias.

- Protocolo de Actuación Integral en Situaciones de Violencia de Género.

- Protocolo de Actuación con Menores en Riesgo.

- Protocolo de Actuación para la Atención de Menores Extranjeros no Acompañados.

- Protocolo de Actuación con Personas sin Techo y Meteorología Extrema.

- Protocolo de Actuación en Catástrofes y Grandes Grupos.

- Protocolo de Actuación con Conductores no Capacitados para Conducir.

\section{Cuestiones para seguir avanzando}

Hoy día, nos encontramos en una posición marcada por el logro de una serie de objetivos importantes, y a la vez, vamos descubriendo el camino por recorrer y mejorar. 


\subsection{Puesta en marcha de los servicios de urgencia sociales}

La administración municipal ha asumido, durante estos años, la responsabilidad y competencia para responder con eficacia ante situaciones imprevistas que sitúan a la ciudadanía en una situación de desprotección biopsicosocial grave. Para ello, ha articulado programas y servicios con capacidad real de intervención, aplicando la metodología y las prestaciones propias de los servicios sociales de forma inmediata.

Las tres capitales han diseñado unos servicios concebidos como mixtos, ya que atienden a la población en general, pero a través de programas dirigidos a situaciones específicas. En síntesis, en las tres capitales disponemos de un servicio creado ad hoc, integrado por profesionales, con una metodología y organización capaces de responder a situaciones de la ciudadanía de forma inmediata, 24 horas al día y 365 días al año.

\subsection{Organización}

El debate de los conceptos de urgencia y emergencia nos resulta un tanto estéril o improductivo. En ambos casos, aceptamos como imprescindible la necesidad de atención inmediata e ineludible por parte de los servicios sociales. Creemos más conveniente, por el contrario, seguir profundizando en la coordinación sistematizada entre los servicios de urgencias sociales y los servicios sociales de base. Entendemos que avanzamos en la dirección correcta, pero con frecuencia nos encontramos con dificultades en relación a qué situaciones deben ser atendidas por unos y otros.

En el caso de los tres municipios, seguramente por razones de rentabilidad y eficacia, nuestro formato 'Servicio Municipal de Urgencias Sociales' desarrolla programas específicos de atención en situaciones de urgencia/emergencia y también otros programas que, aunque próximos al concepto, pueden desarrollarse desde otros formatos con igual validez (gestión de casos que precisan alta intensidad, gestión de recursos para personas víctimas de violencia de género, acceso a prestaciones básicas). No existen razones especiales: es operativo, nos va bien. En realidad, es un formato flexible y versátil.

\subsection{Intervención integral y coordinada}

Todo lo avanzado en procedimientos de actuación coordinada -especialmente con servicios sanitarios, Seguridad Ciudadana, Protección Civil y Urbanismonos lleva a concluir lo importante de que esta intervención sea integral y coordinada con otras áreas y servicios municipales y, en su caso, con otras instituciones (Gobierno Vasco, diputaciones, juzgados).

\subsection{Participación en los planes de emergencia}

La intervención de los servicios sociales (funciones y prestaciones) se manifiesta fundamental en la cobertura de necesidades y el logro del bienestar de la ciudadanía que vive una situación de emergencia individual o colectiva. Por lo tanto, la acción social municipal ha de participar en la elaboración, el desarrollo y la evaluación de los planes de emergencia, junto con otros departamentos municipales intervinientes.

\subsection{Inmediatez en el diagnóstico, la prescripción y la provisión de recursos}

La capacidad de realizar un diagnóstico de forma inmediata ha de ir acompañada de la prescripción y provisión de los recursos necesarios, igualmente de forma inmediata. Todo esto es fundamental en la atención de 24 horas al día y 365 días al año, especialmente en situaciones como las siguientes:

- Personas que viven solas y se accidentan en su domicilio: asistencia para la higiene personal, la limpieza de habitación y la preparación de alimento, mediante un servicio de ayuda a domicilio u otras fórmulas, en horario de tarde o noche, y de forma inmediata.

- Soledad sobrevenida por desaparición temporal o definitiva de la persona cuidadora: ingreso inmediato en una vivienda comunitaria o residencia para personas mayores, o un centro residencial para personas con discapacidad.

- Convalecencia de una persona sin techo: ingreso inmediato en una plaza residencial.

- Familia con menores sin hogar: alojamiento.

\subsection{Cartera y Mapa de Servicios Sociales}

En nuestra ley $12 / 2008$, de Servicios Sociales, se establece como derecho subjetivo el acceso a los servicios sociales. En ella, se contemplan las situaciones de emergencia como un ámbito de actuación propio (artículo 6) y se establece asimismo que las personas que se encuentren en la CAPV podrán acceder a aquellos servicios y prestaciones que sean definidos como de urgencia social en la Cartera de Prestaciones y Servicios Sociales (artículo 3.3), sin exigencias de plazos previos de empadronamiento.

Por consiguiente, es preciso, y así lo esperamos los y las profesionales, que la Cartera y el Mapa de Servicios Sociales ofrezcan respuestas adecuadas, que permitan un acceso igualitario de la ciudadanía a los servicios sociales en situaciones de emergencia y superar las desigualdades territoriales a las que estamos acostumbrados. Entre otras, creemos que son cuestiones importantes: 
- El acceso a plazas residenciales para las urgencias sociales, tal y como recoge la disposición adicional séptima.

- Definir cuáles son los servicios y prestaciones definidos como de urgencia social (en art. 3.3). Sería deseable y conveniente que, además, se establezcan plazos de atención y ratios de plazas residenciales.

- Determinar qué incidencia puede tener lo expuesto en el art. 60.2: las prestaciones de primera acogida de las demandas, la valoración, el diagnóstico y la orientación serán siempre de gestión pública directa.

- Regular el acceso a prestaciones básicas (alimento, alojamiento, vestido) en relación al derecho subjetivo y a las prestaciones de urgencia.

- Integrar los servicios sociales de atención primaria en las grandes emergencias.

- Integrar el voluntariado: posibilidad de convenios con agencias locales de voluntariado y colegios profesionales (de trabajo social, de psicólogos). 